\title{
MRI T2 Mapping of Knee Articular Cartilage Using Different Acquisition Sequences and Calculation Methods at 1.5 Tesla
}

\author{
Mokhtar Mars $^{\mathrm{a}} \quad$ Mouna Chelli $^{\mathrm{b}} \quad$ Zeineb Tbini $^{\mathrm{a}} \quad$ Fethi Ladeb $^{\mathrm{b}}$ Souha Gharbi ${ }^{\mathrm{a}}$ \\ aTunis University EL Manar, Higher Institute of Medical Technologies of Tunis, Research Laboratory of Biophysics \\ and Medical Technologies, Tunis, Tunisia; ${ }^{b}$ Tunis University EL Manar, Faculty of Medicine of Tunis, Department of \\ Radiology, Kassab Institute of Orthopedics, Ksar Saïd, Tunis, Tunisia
}

\section{Significance of the Study}

- Magnetic resonance imaging T2 mapping of the knee articular cartilage depends not only on acquisition techniques but also on calculation methods. This study focuses on the different $\mathrm{T} 2$ values that have to be considered when interpreting T2 values of knee articular cartilage. This study also shows that Turbo Gradient Spin Echo sequence could be used for T2 mapping with the advantage of high in-plane resolution in a relatively short scanning time.

\section{Keywords}

Knee cartilage $\cdot$ MRI osteoarthritis · T2 mapping ·

Calculation methods

\begin{abstract}
Objective: This study aims to determine how magnetic resonance imaging (MRI) acquisition techniques and calculation methods affect T2 values of knee cartilage at 1.5 tesla and to identify sequences that can be used for high-resolution T2 mapping in short scanning times. Materials and Methods: This study was performed on phantom and 29 patients who underwent MRI of the knee joint at 1.5 tesla. The protocol includes T2 mapping sequences based on Single-Echo Spin Echo (SESE), Multi-Echo Spin Echo (MESE), Fast Spin Echo (FSE) and Turbo Gradient Spin Echo (TGSE). The T2 relaxation times were quantified and evaluated using three calculation methods (Maplt, Syngo Offline and mono-exponential fit).
\end{abstract}

signal-to-noise ratios (SNR) were measured in all sequences. All statistical analyses were performed using the $\boldsymbol{t}$-test. $\boldsymbol{R} \boldsymbol{e}$ sults: The average T2 values in phantom were $41.7 \pm 13.8 \mathrm{~ms}$ for SESE, $43.2 \pm 14.4 \mathrm{~ms}$ for MESE, $42.4 \pm 14.1 \mathrm{~ms}$ for FSE and $44 \pm 14.5 \mathrm{~ms}$ for TGSE. In the patient study, the mean differences were $6.5 \pm 8.2 \mathrm{~ms}, 7.8 \pm 7.6 \mathrm{~ms}$ and $8.4 \pm 14.2 \mathrm{~ms}$ for MESE, FSE and TGSE compared to SESE, respectively; these statistical results were not significantly different $(p>0.05)$. The comparison between the three calculation methods showed no significant difference $(p>0.05)$. The $t$-test showed no significant difference between SNR values for all sequences. Conclusion: T2 values depend not only on the sequence type but also on the calculation method. None of the sequences revealed significant differences compared to the SESE reference sequence. TGSE with its short scanning time can be used for high-resolution T2 mapping.

(C) 2018 The Author(s)

Published by S. Karger AG, Basel

\begin{tabular}{ll}
\hline KARGER & $\begin{array}{l}\text { Ko 2018 The Author(s) } \\
\text { Published by S. Karger AG, Basel Oparger }\end{array}$ \\
$\begin{array}{l}\text { E-Mail karger@karger.com } \\
\text { Www.karger.com/mpp }\end{array}$ & $\begin{array}{l}\text { This is an Open Access article licensed under the Creative Commons } \\
\text { Attribution-NonCommercial-4.0 International License (CC BY-NC) } \\
\text { (http://www.karger.com/Services/OpenAccessLicense), applicable to } \\
\text { the online version of the article only. Usage and distribution for com- } \\
\text { mercial purposes requires written permission. }\end{array}$
\end{tabular}

Mokhtar Mars

29 Rue Imam Chafai La Petite Ariana

Jaafar, 2083 Tunis (Tunisia)

E-Mail mokhtar.mars-mms@ topnet.tn 


\section{Introduction}

Knee osteoarthritis (OA) is characterized by progressive loss of articular cartilage. It is the most common form of arthritis and the leading cause of disability for adults [1]. Magnetic resonance imaging (MRI) is the technique of choice for the assessment of articular cartilage pathologies due to its high soft tissue contrast, better spatial resolution and the possibility to acquire and reconstruct in different planes [2]. Conventional MRI sequences are used to evaluate anatomy and detect morphological changes of the knee cartilage. Both radiography and morphologic MRI of the knee are not able to detect early stages of $\mathrm{OA}$, and the response to therapy after cartilage repair procedures [3, 4].

While different options exist for the treatment of OA, the efficiency and the implementation of treatment strategies to slow or to stop progression of the disease depend on early and accurate detection of the stage of OA [5].

In the earliest stage of OA and before morphological changes occur, degenerative changes in the cartilage are related to loss of proteoglycan and deterioration of the collagen network within the cartilage. The cartilage becomes less elastic, allowing increased mobility of water and consequentially increased levels of $\mathrm{H}_{2} \mathrm{O}$ proton content.

Quantitative MRI mappings, which are used to translate MRI relaxation times into quantitative values of tissues [6], are able to show subtle changes in cartilage composition in early stages even before structural changes appear [7]. The available mappings for cartilage tissue are T1 mapping, T2 mapping and T2-star (T2*) mapping but the most commonly used is T2 mapping which can evaluate the status of the cartilage matrix and identify biochemical changes associated with the early stages of OA $[8,9]$. Several studies have demonstrated that T2 mapping is useful for the detection of early stages of matrix degeneration [10-13].

T2 mapping can be calculated using T2 sequence with different TEs. Many sequences have been used for the calculation of $\mathrm{T} 2$ relaxation Time. The most fundamental sequences used are spectroscopy and SingleEcho Spin Echo (SESE). Other 2D sequences have been used, such as Multi-Echo Spin Echo (MESE), Fast Spin Echo (FSE) and Turbo Gradient Spin Echo (TGSE). 3D sequences have also been used, such as Double Echo Steady State (DESS) sequence, where data from two acquisitions with different spoiler gradient areas and flip angles were used to simultaneously estimate the T2 of each pixel [14]. Synthetic MRI is another quantitative method in which a single saturation recovery turbo spin echo sequence is used to estimate T2 transverse relaxation [15].

The cartilage is a thin structure and due to its short $\mathrm{T} 2$, a very poor signal-to-noise ratio (SNR) and long scan time are necessary when using spectroscopy or SESE. Those features prevent the use of either sequence in routine clinical practice. To overcome these limitations, three 2D sequences have been developed: MESE, FSE and TGSE. The MESE sequence measures many echoes during one Repetition Time (TR). The FSE acquires many phases in one TR. The TGSE sequence combines gradient echo and spin echo imaging. The spin echo gives the T2 contrast, and the gradient echo determines the image resolution.

The first goal of this study was to determine how acquisition techniques and calculation methods affect T2 values at 1.5 tesla and the second was to identify a fast sequence that can be used for high-resolution T2 mapping of the different layers of articular cartilage in a short scanning time.

\section{Materials and Methods}

This prospective study was approved by our institutional review board and patient consent was obtained. The study was performed on phantom and patients.

\section{Phantom Study}

To compare T2 maps, we designed a phantom from a sodium chloride solution. To this solution, we added different concentrations of contrast agent (Dotarem, Guerbet, USA) to obtain a range of $\mathrm{T} 2$ values similar to that found in the articular cartilage of the knee $(\sim 20-70 \mathrm{~ms})$. In total, the phantom was composed of six samples.

\section{Patient Study}

Twenty-nine subjects underwent MRI of the knee joint at 1.5 tesla. Subjects included 17 asymptomatic patients (9 males and 8 females; mean age: $32.7 \pm 8.8$ years) and 12 symptomatic patients (11 males and 1 female; mean age: $42.66 \pm 11.3$ years) with different pathologies such as $\mathrm{OA}$ and meniscus lesions. Special attention was paid to ensure that all patients were well fixed with the joint space in the middle of the coil and that the knee was extended in the coil. Three measurements were excluded due to patient's movements.

\section{Image Acquisition}

Examinations were performed at 1.5 tesla MRI system (Magnetom Aera, Siemens Erlangen, Germany) with a dedicated 15-element knee coil. The imaging protocol for the patient study included morphological and biochemical sequences.

To standardize acquisition parameters between sequences, we adhered to the following technical aspects. The spin echo sequence family suffers from steady-state effects (i.e., different $\mathrm{T} 1$ 

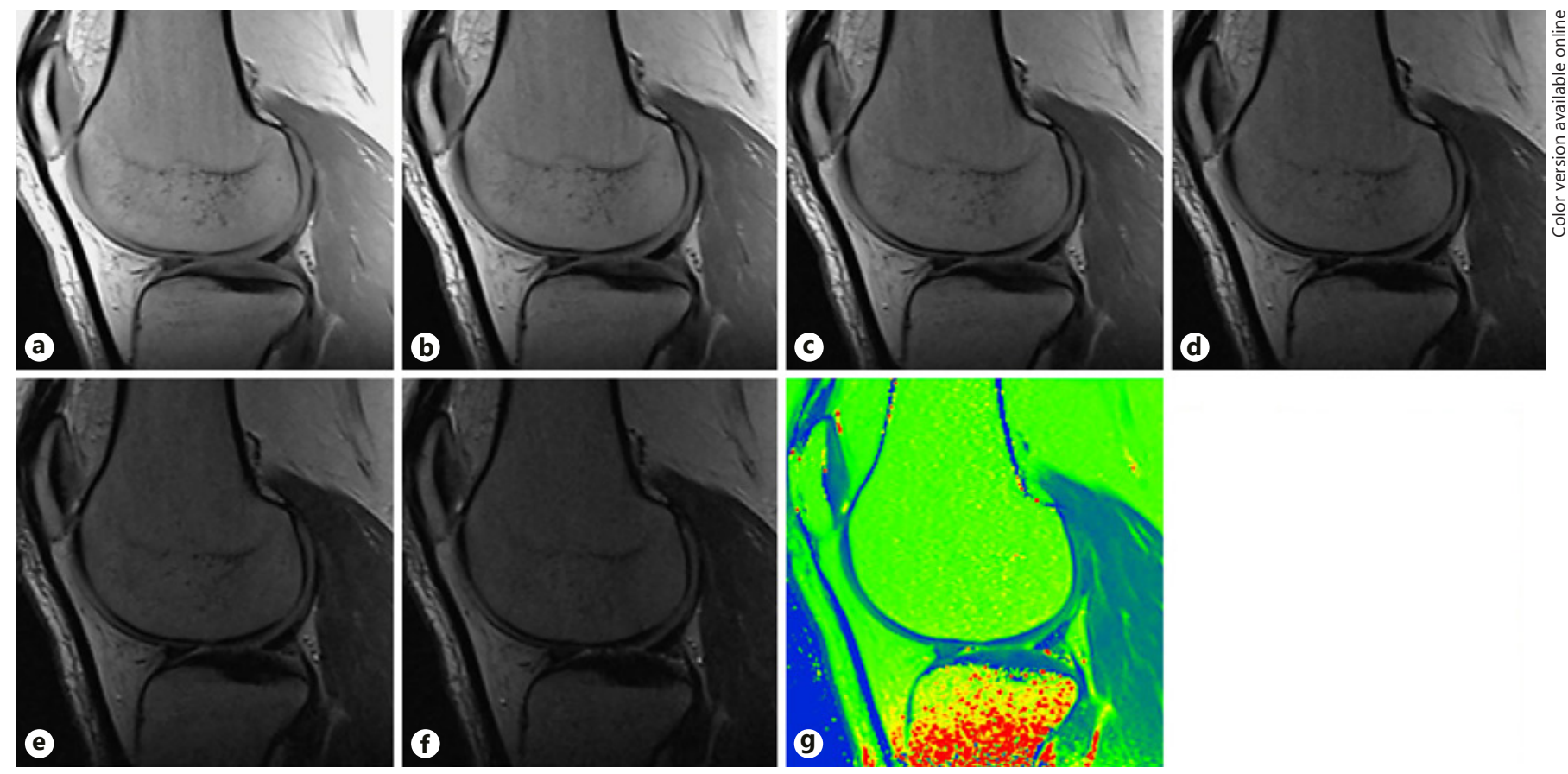

Fig. 1. a-f Images from the SESE reference sequence acquired with different TEs as well as the resultant T2 map. $\mathbf{a} \mathrm{TE}=12 \mathrm{~ms}$. $\mathbf{b} \mathrm{TE}=$ $24 \mathrm{~ms}$. $\mathbf{c ~ T E}=36 \mathrm{~ms}$. d TE $=50 \mathrm{~ms}$. e TE $=60 \mathrm{~ms}$. f TE $=70 \mathrm{~ms}$. g T2 map.

Table 1. Scanning parameters of sequences used for T2 mapping

\begin{tabular}{|c|c|c|c|c|c|}
\hline Parameter & \multicolumn{5}{|l|}{ Sequence } \\
\hline Echo time, ms & $20-30-40-50-60$ & $12-24-36-50-60-70$ & $12.5-25-37.5-50-62.5$ & $19-50-69$ & $25-50-70$ \\
\hline Field of view, mm & n.a. & $160 \times 160$ & $160 \times 160$ & $160 \times 160$ & $160 \times 160$ \\
\hline Bandwidth/pixel, $\mathrm{Hz}$ & 1,200 & 150 & 150 & 310 & $454-173-292$ \\
\hline Slice gap, mm & n.a. & 3 & 3 & 3 & 3 \\
\hline Slices, $n$ & n.a. & 11 & 11 & 11 & 11 \\
\hline Excitations, $n$ & 35 & 1 & 1 & 1 & 1 \\
\hline Acquisition time, min:s & $1: 00$ & $16: 54$ & $2: 50$ & $2: 08$ & $1: 54$ \\
\hline
\end{tabular}

n.a., not applicable.

weightings) in the images. To minimize the T1 contribution to the image contrast, we used a TR of $1,500 \mathrm{~ms}$ [16]. We chose the sagittal plane because it allows the evaluation of articular cartilage in a direction perpendicular to the majority of the weight forces acting on the joint [17]. We chose a bandwidth of approximately $220 \mathrm{~Hz} /$ pixel, which corresponds to a chemical shift of 1 pixel in 1.5 tesla. To compare the sequences in an efficient manner, we used the same geometrical parameters (same field of view, slice thickness and matrix). Images acquired with SESE sequence as well as the T2 map result are shown in Figure 1. We used a common TE value of $50 \mathrm{~ms}$ in all sequences for comparison purposes. For the phantom study, we used the same sequences as for the patient and we added the spectroscopy sequence. For the spectroscopy, we used the STEAM technique with a volume of interest (VOI) of $16 \mathrm{~mm} \times 16 \mathrm{~mm} \times 16 \mathrm{~mm}$. The details of scanning parameters for all sequences are listed in Table 1. 
Fig. 2. Comparison of the curves: signals between different sequences as a function of TE in phantom study. a TE as a function of absolute values. $\mathbf{b}$ TE as a function of normalized values.

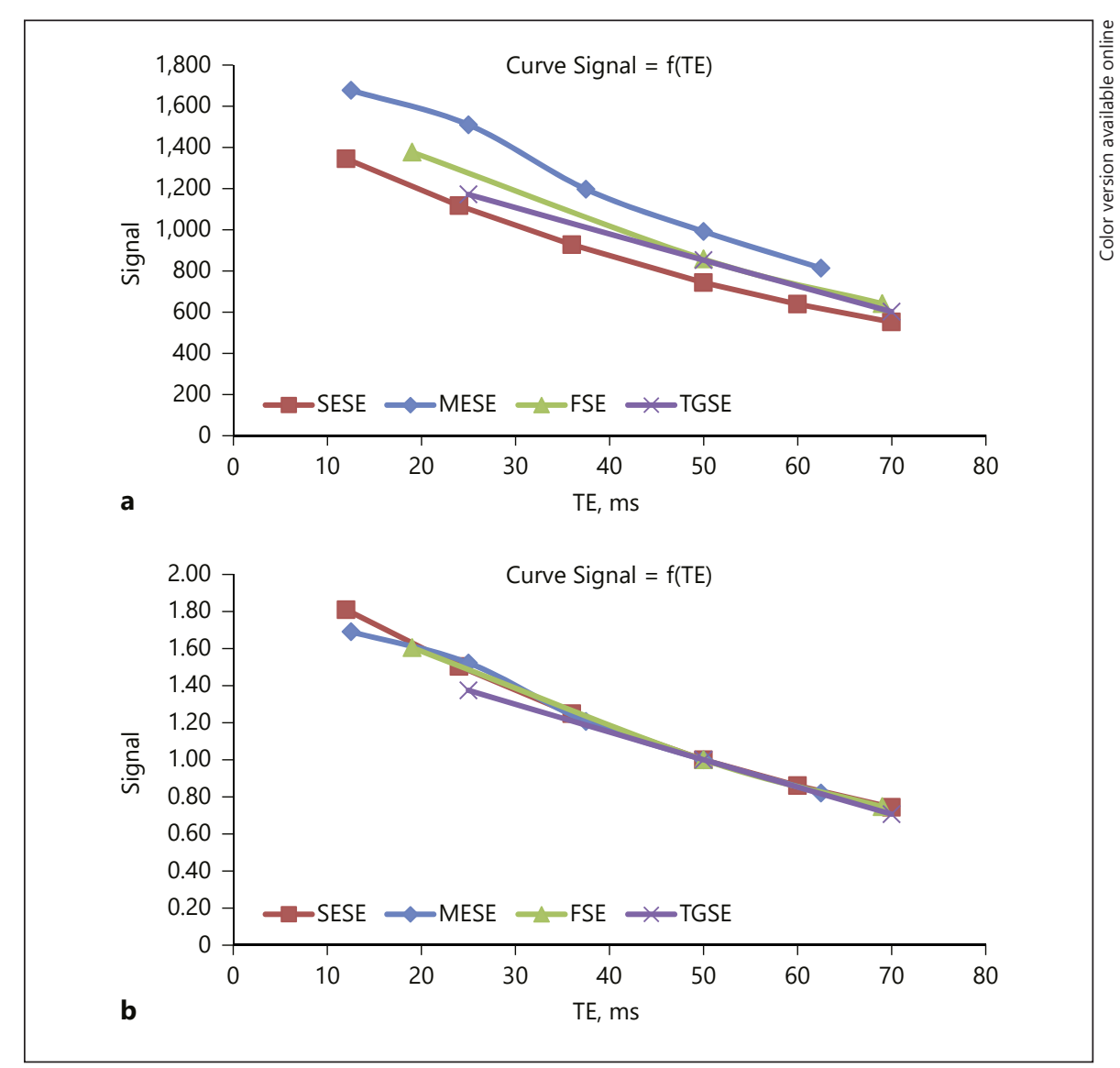

\section{Statistical Analysis}

For phantom evaluation, the region of interest (ROI) was drawn in the center of each sample. To compare all sequences with the SESE reference sequence, we used the same mono-exponential fitting calculation method.

For patient assessment, the inner margin of the meniscus was used as a marker for determining the anterior and posterior borders of the weight-bearing cartilage on the sagittal MR images [17]. The femoral cartilage was segmented into anterior, median and posterior. A total of six ROIs were drawn in the internal and external sagittal parts of the knee. We did not include the superficial zone to avoid the chemical shift artifact.

T2 maps were obtained using three techniques. The first technique is a pixel-wise, mono-exponential non-negative leastsquare (NNLS) fit analysis (MapIt, Siemens Medical Solutions, Erlangen, Germany). The second technique is the offline calculation using the Syngo software from Siemens, and the third technique is based on mono-exponential fitting using the equation $\mathrm{S}(\mathrm{t})=\mathrm{S}_{0}{ }^{*} \exp (-\mathrm{t} / \mathrm{T} 2)$. In the MapIt analysis, the first echo was excluded from the fit to reduce error resulting from signals produced by the stimulated echo.

The MR signal depends on type and parameters of the sequence. T2 relaxation curve follows an exponential decay. The MR signal depends mostly on the TE and better signals would be obtained using short TE. To compare the SNR between sequences in an efficient way, we used a common TE value of $50 \mathrm{~ms}$. The SNR was calculated as the ratio of the mean cartilage region signal divided by the signal of the background.

We compared all sequences to the SESE reference sequence. We performed linear regression on each of these plots. The slope of each regression line was used as an indicator of the dynamic range of T2 relaxation time in each sequence. We calculated the mean $\mathrm{T} 2$ values and standard deviations from different sequences for phantom and patients. All statistical analyses were performed using the $t$-test (SPSS software version 15 @ 2006). For all statistical tests, $p$ value less than 0.05 was considered significant.

\section{Results}

\section{Phantom Study}

In the phantom study, we applied our protocol to six samples. The results showed that all sequences demonstrated strong fits with $\mathrm{R}^{2}>0.98$. Both FSE and SESE exhibited a high coefficient of determination with an $\mathrm{R}^{2}$ min of 0.999 , compared with 0.990 obtained with TGSE and 0.986 obtained with MESE.

The results revealed mean $\mathrm{T} 2$ values in the phantom of $41.7 \pm 13.8 \mathrm{~ms}$ for SESE, $43.2 \pm 14.4 \mathrm{~ms}$ for MESE, $42.4 \pm$ 
Table 2. Slopes and coefficients of determination $\mathrm{R}^{2}$ in all regions of interest (ROIs) of sequences compared to the SESE reference sequence

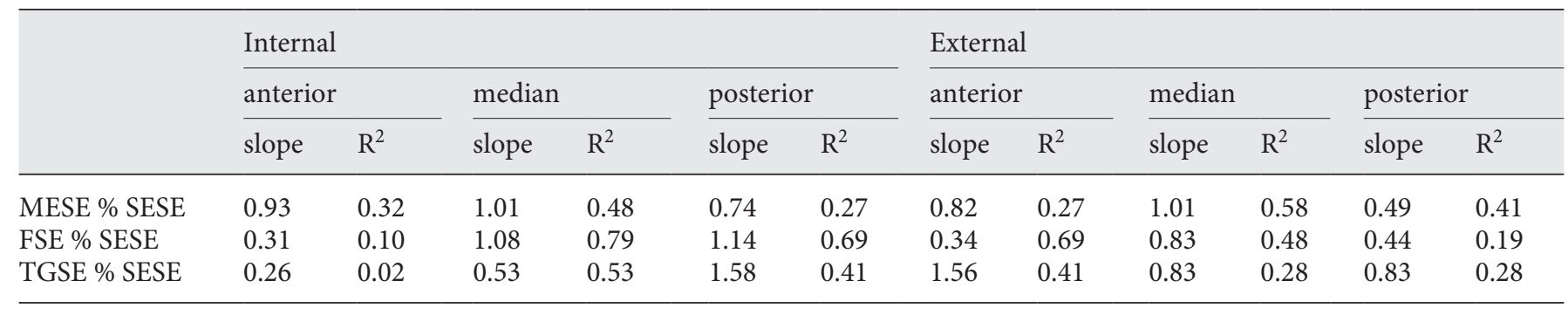

$14.1 \mathrm{~ms}$ for FSE and $44 \pm 14.5 \mathrm{~ms}$ for TGSE. Our results showed a mean difference of $1.6 \pm 1 \mathrm{~ms}$ and mean percent difference of $3.7 \pm 2 \%$ for MESE compared to SESE. The comparison between FSE and SESE revealed a mean difference of $0.7 \pm 0.8 \mathrm{~ms}$ and a mean percent difference of $1.7 \pm 2 \%$. The mean difference between TGSE and SESE was $2.4 \pm 0.8 \mathrm{~ms}$ with a mean percent difference of $5.7 \pm$ $1 \%$. For STEAM, our study was limited to one sample (sample 6). The mean T2 values of sample 6 were $63.5 \mathrm{~ms}$, $64.9 \mathrm{~ms}, 67 \mathrm{~ms}, 65.4 \mathrm{~ms}$ and $68 \mathrm{~ms}$ using STEAM spectroscopy, SESE, MESE, FSE and TGSE, respectively.

The slopes of the plot lines between all sequences compared to our SESE reference sequence were 1.04, 1.017 and 1.048 with correlation coefficients $\mathrm{R}^{2}=0.996,0.996$ and 0.999 for MESE, FSE and TGSE, respectively.

Statistical results using the $t$-test showed no significant difference between T2 values when TGSE, MESE and FSE were compared with SESE reference sequence with $p$ values equal to 1,1 and 0.998 respectively.

MR signal and contrast depend strongly on TE. To compare the graph of all sequences, we used the same TE of $50 \mathrm{~ms}$ in all sequences (Fig. 2a). A qualitative comparison between images from different sequences acquired at the same TE of $50 \mathrm{~ms}$ shows that images acquired with MESE and FSE are smoother and that cartilage-meniscus contrast is better with SESE and TGSE. Then, we normalized all values using the same TE of $50 \mathrm{~ms}$ as a reference by calculating the ratio of the signal intensity of each TE divided by the signal intensity of $\mathrm{TE}=50 \mathrm{~ms}$. Results are shown in Figure 2b.

\section{Patient Study}

In the present patient study, the mean difference between MESE and SESE reference sequence was $6.5 \pm 8.2$ ms with a percent difference of $13.5 \pm 16 \% \mathrm{~ms}$. The comparison between FSE and SESE reference sequence showed a mean difference of $7.8 \pm 7.6 \mathrm{~ms}$ with a percent difference of $15.5 \pm 13 \%$ ms. The calculated mean difference of TGSE T2 values compared to SESE reference sequence was $8.4 \pm 14.2 \mathrm{~ms}$ with a percent difference of 5.6 $\pm 21 \% \mathrm{~ms}$. Table 2 present the comparison results of the coefficient of determination $\mathrm{R}^{2}$ and the slope between SESE reference sequence and MESE, FSE and TGSE, respectively.

Statistical results using the $t$-test showed no significant difference between T2 values when TGSE, MESE and FSE were compared to the SESE reference sequence. $p$ values were $0.267,0.704$ and 0.726 , respectively.

In the anterior ROI, SNR values were 12.65, 9.86, 14.57 and 12.5 for SESE, MESE, FSE and TGSE sequences, respectively. In the median ROI, they were 12.63, 9.84, 14.54 and 12.3 for SESE, MESE, FSE and TGSE, respectively, and in the posterior ROI, they were 12.67, 9.88, 14.56 and 11.85 for SESE, MESE, FSE and TGSE, respectively. The comparison of SESE, MESE, FSE and TGSE in terms of SNR showed no significant difference with $p$ values of $0.953,0.698$ and 0.699 , respectively.

\section{Evaluation Methods}

T2 values calculated using MapIt were less than T2 values calculated with mono-exponential fitting function. The mean difference was $3.8 \pm 2.9 \mathrm{~ms}$ and the maximum percent difference was $9.05 \pm 7.2 \%$ with no significant difference $(p=0.956)$. Compared to the calculation using Syngo Offline, the mono-exponential fitting function method produced lower T2 values with a mean difference of $2 \pm$ $4.6 \mathrm{~ms}$ and maximum percent difference of $3.4 \pm 8.3 \%$ with no significant difference $(p=0.956)$. T2 values calculated with the mono-exponential fitting function method using non-stimulated echo (without using the first echo) were increased compared to $\mathrm{T} 2$ values calculated using all echoes in the MESE sequence, with a mean difference of $2 \pm 2.5 \mathrm{~ms}$ and a maximum percent difference of $4.1 \pm 4.5 \%$. Results revealed no significant difference $(p=0.956)$. 


\section{Discussion}

The main goal of this study was to determine how acquisition techniques and calculation methods affect the T2 values at 1.5 tesla. This study compared MESE, FSE and TGSE to SESE reference sequence in order to identify a fast sequence that can be used for high-resolution T2 mapping in short scanning time.

The most fundamental sequences used for T2 mapping are spectroscopy and SESE. Spectroscopy sequence suffers from partial volume and low SNR. Thus, this sequence is used only with phantom studies. The acquisition time of SESE sequence is relatively long, making its application in routine clinical practice very time consuming and difficult to implement.

For the MESE sequence, results showed that the mean difference of MESE compared to SESE reference sequence was increased by $3.7 \pm 2 \mathrm{~ms}$ in the phantom study and $6.5 \pm 8.2 \mathrm{~ms}$ in the patient study. Our results are in agreement with previous studies. Maier et al. [18] observed an increase by $10-13 \%$ of the $\mathrm{T} 2$ relaxation time on phantom using MESE with slice-selective refocusing pulses at 1.5 tesla compared to SESE. The study also showed increases of T2 values by up to $48 \%$ with MESE compared to SESE [18]. In a previous study conducted on 10 healthy volunteers using 3-tesla system, the MESE sequence showed an increase by $24.8 \%$ of the cartilage T2 relaxation times compared to spin echo sequence [19]. The increased T2 values in these studies could be explained by the stimulated echoes generated in the multiecho sequences.

For FSE, our phantom study indicated good fitting with $\mathrm{R}^{2}=0.999$ and in the patient study, the comparison of the plot of FSE with SESE revealed a coefficient of determination $\mathrm{R}^{2}=0.52$. A previous experimental study conducted by Matzat et al. [11] at 3 tesla showed that measurements from all sequences demonstrated strong fits $\left(\mathrm{R}^{2}>0.8\right)$ in the agar phantom and a coefficient of determination $\mathrm{R}^{2}=0.64$ for patients. The difference could be explained by the difference in phantom composition and the fact that $\mathrm{T} 2$ relaxation time is reduced in high magnetic field [20]. The comparison between FSE and SESE on patients showed positive mean and percent difference, which indicate that T2 values obtained with FSE were increased compared to SESE.

In another previous study performed at 3 tesla MRI system with 8 volunteers, measurements from the FSE sequence were $25-38 \%$ higher than those from all other sequences on phantom and patient articular cartilage [11]. Another study showed that T2 relaxation times in- crease as the echo train length of the FSE sequence increases [19]. This phenomenon can be explained by the presence of stimulated echoes throughout the echo train that result from imperfect $180^{\circ}$ refocusing pulses. Residual longitudinal magnetization between echoes results in partial T1-weighting, leading to an elevation in T2 relaxation time estimation.

The comparison of TGSE sequence with SESE in patient study revealed small coefficients of determination $\mathrm{R}^{2}$ and increased T2 values. Quaia et al. [8] found that gradient and spin-echo sequence (GRASE) provided T2 values slightly lower than those obtained by TSE sequence in most patients (mean difference $\pm \mathrm{SD}, 1.81 \pm 3.63 \mathrm{~ms}$ ). This difference may be due to the presence of $\mathrm{T} 2 *$ decay in the GRASE sequence. The authors observed artifacts such as the chemical shift, image distortion and signal loss [8].

For SNR evaluation, results revealed no significant difference with $p>0.05$. Since we used the same geometrical values for all sequences, the choice of optimum sequence will depend mostly on number of echoes and scan time. Statistical results using the $t$-test revealed no significant difference $(p>0.05)$ for FSE, SEME and TGSE compared to SESE reference sequence, respectively.

In a previous study, it was demonstrated that $\mathrm{T} 2$ values of the knee femoral cartilage were significantly lower on post-contrast images compared to pre-contrast images $(35.3 \pm 9.2 \mathrm{~ms}$ vs. $29.9 \pm 8.2 \mathrm{~ms}, p<0.01)$ [21]. This has to be taken into account because most T2 mapping is performed without the use of intravenous contrast agents. Another study indicated that intra-articular injection of Gd-DTPA2 had little effect on T2* values of femoral cartilage [22].

One of the disadvantages of T2 mapping is the susceptibility to the magic angle effect, in which T2 values may be artificially elevated in certain regions according to the orientation of cartilage in relation to the main magnetic field. The T2 values were greater when the cartilage regions were oriented at $55^{\circ}$ to $\mathrm{B}_{0}$ and were the lowest in subregions oriented at $180^{\circ}\left(0^{\circ}\right)$ to $\mathrm{B}_{0}$ [23]. However, this effect should not impact results between study populations as long as the subjects are positioned in the same manner in the magnet and the evaluated regions of the cartilage are the same for all patients.

Therefore, when interpreting T2 mapping results, it is important to use caution because the $\mathrm{T} 2$ value changes according to the strength of the magnetic field $\mathrm{B}_{0}$. Shorter T2 values were found at higher field strengths [24]. In addition, the sequence type, coil architecture and calculation method of T2 mapping affect the T2 result [11]. 


\section{Calculation Methods}

Our data show that $\mathrm{T} 2$ values calculated using Inline calculation (MapIt) were less than T2 values calculated with mono-exponential fitting function. This finding is explained by the fact that MapIt excludes the first stimulated echo from the calculation. Compared to Syngo Offline, the mono-exponential fitting method showed decreased T2 values. The Syngo Offline method included a noise offset correction, which will discard supposed "noisy" signal points for fitting of the T2 curve. The selection of the noise value and the difference of the fitting method may explain this variation.

A previous study demonstrated that traditional fitting methods showed poor T2 accuracy for low T2 values, whereas the noise-corrected fitting methods demonstrated very good accuracy for all T2 values. Therefore, the sensitivity of T2 mapping to detect pathology in cartilage tissue may be improved by using an adequate fitting method [25]. Koff et al. [26] found that linear, weighted and non-linear fitting algorithms resulted in significantly different T2 values $(p<0.0001)$. The non-linear calculation demonstrated the lowest T2 values, the linear calculation resulted intermediate $\mathrm{T} 2$ values and the weighted calculation resulted in the highest T2 values [26].

T2 mapping calculation can be realized using either mono- or bi-exponential fitting. It was reported that $\mathrm{T} 2$ relaxation time based on bi-exponential in the human knee cartilage has the advantage of detecting short and long components related to the tightly bound and loosely bound macromolecular water compartments which may increase the specificity for detection of early OA [27].

\section{Limitations}

Some limitations of this study should be noted. First, it is necessary that these findings be validated in a larger group of patients. Second, the phantom used in our study has the same $\mathrm{T} 2$ relaxation time as the cartilage but not the same T1 relaxation value and the same property of articular cartilage. Another limitation of our study involves possible partial volume effects. These effects were reduced in our study because the superficial zone was not included when ROIs were drawn. This limitation could be solved by increasing the acquisition matrix to obtain an adequate in-plane spatial resolution, but this protocol will increase the scan time, which remains challenging for in vivo cartilage imaging. We did not subdivide the cartilage into subcompartments because the reduction of ROI areas will reduce precision and reproducibility. The reproducibility of our results on the position of the ROIs might affect the T2 values found but the problem is reduced since we copy the same ROIs in different sequences and also because we use the same pixel size. Another point is the stability of the phantom composition which was not studied. The major limitation of the study is that we used 2D sequences instead of 3D sequences which offer improved SNR, thin slices and the possibility to reformat in other planes. But those 3D sequences are not available as standard in any MRI system. Another limitation is that we evaluated T2 relaxation times only in the femoral cartilage. It would be better to evaluate the patellar and tibial cartilage as well. However, our aim was to compare $\mathrm{T} 2$ values depending on the sequence type and not $\mathrm{T} 2$ values in different articular cartilage regions.

\section{Conclusions}

Our findings are of importance with regard to the process of optimization of practice in MR examinations. MRI acquisition techniques and calculation methods affect T2 values of knee articular cartilage at 1.5 tesla. For accurate clinical applications of T2 mapping, spatial variation of T2 relaxation times may be considered rather than exclusively using the absolute values. TGSE can be used for T2 calculation with the advantage of high resolution in an acceptable time frame compared to other sequences.

\section{Acknowledgements}

Special thanks to Professor Ali Mrabet for his support in the statistical evaluation.

\section{References}

MRI T2 Mapping of the Knee Articular

Cartilage at 1.5 Tesla
1 Baum T, Joseph GB, Karampinos DC, et al: Cartilage and meniscal T2 relaxation time as non-invasive biomarker for knee osteoarthritis and cartilage repair procedures. Osteoarthritis Cartilage 2013;21:1474-1484.

2 Garry EG, Christina AC, Seungbum K, et al: Recent advances in MRI of articular cartilage. Am J Roentgenol 2009;193:628-638.
3 Chu CR, Williams AA, Coyle CH, et al: Early diagnosis to enable early treatment of pre-osteoarthritis. Arthritis Res Ther 2012;14:212.

4 Hunter DJ, Zhang W, Conaghan PG, et al: Systematic review of the concurrent and predictive validity of MRI biomarkers in OA. Osteoarthritis Cartilage 2011;19:557-588. 
5 Surowiec RK, Lucas EP, Fitzcharles EK, et al: $\mathrm{T} 2$ values of articular cartilage in clinically relevant subregions of the asymptomatic knee. Knee Surg Sports Traumatol Arthrosc 2013; 22:1404-1414.

6 Wilson KJ, Surowiec RK, Johnson NS, et al: T2* Mapping of peroneal tendons using clinically relevant subregions in an asymptomatic population. Foot Ankle Int 2017;38:677-683.

7 Lazik A, Theysohn JM, Geis C, et al: 7 Tesla quantitative hip MRI: T1, T2 and T2* mapping of hip cartilage in healthy volunteers. Eur Radiol 2016;26:1245-1253.

8 Quaia E, Toffanin R, Guglielmi G, et al: Fast T2 mapping of the patellar articular cartilage with gradient and spin-echo magnetic resonance imaging at $1.5 \mathrm{~T}$ : validation and initial clinical experience in patients with osteoarthritis. Skeletal Radiol 2008;37:511-517.

9 Phan CM, Link TM, Blumenkrantz G, et al: MR imaging findings in the follow-up of patients with different stages of knee osteoarthritis and the correlation with clinical symptoms. Eur Radiol 2006;16:608-618.

10 White LM, Sussman MS, Hurtig M, et al: Cartilage T2 assessment: differentiation of normal hyaline cartilage and reparative tissue after arthroscopic cartilage repair in equine subjects. Radiology 2006;241:407-414.

11 Matzat SJ, McWalter EJ, Kogan F, et al: T2 relaxation time quantitation differs between pulse sequences in articular cartilage. J Magn Reson Imaging 2015;42:105-113.

12 Crema MD, Roemer FW, Marra MD, et al: Articular cartilage in the knee: current MR imaging techniques and applications in clinical practice and research. Radiographics 2011; 31:37-61.
13 Quatman CE, Hettrich CM, Schmitt LC, et al: The clinical utility and diagnostic performance of magnetic resonance imaging for identification of early and advanced knee osteoarthritis: a systematic review. Am J Sports Med 2011;39:1557-1568

14 Staroswiecki E, Granlund KL, Alley MT, et al: Simultaneous estimation of T2 and ADC in human articular cartilage in vivo with a modified 3D DESS sequence at $3 \mathrm{~T}$. Magn Reson Med 2012;67:1086-1096.

15 Park S, Kwack KS, Lee YJ, et al: Initial experience with synthetic MRI of the knee at 3T: comparison with conventional T1-weighted imaging and T2 mapping. Br J Radiol 2017;90: 1080-20170350.

16 Koff MF, Amrami KK, Kaufman KR: Clinical evaluation of T2 values of patellar cartilage in patients with osteoarthritis. Osteoarthritis Cartilage 2007;15:198-204.

17 Jang GC, Jae CL, Hyun JK, et al: Comparison of MRI T2 relaxation changes of knee articular cartilage before and after running between young and old amateur athletes. Korean J Radiol 2012;13:594-601.

18 Maier CF, Tan SG, Hariharan H, et al: T2 quantitation of articular cartilage at $1.5 \mathrm{~T}$. J Magn Reson Imaging 2003;17:358-364.

19 Pai A, Li X, Majumdar S: A comparative study at $3 \mathrm{~T}$ of sequence dependence of $\mathrm{T} 2$ quantitation in the knee. Magn Reson Imaging 2008; 26:1215-1220.
20 Goetz H, Sebastian A, Stefan Z, et al: Biochemical (T2, T2* and magnetisation transfer ratio) MRI of knee cartilage: feasibility at ultra-high field (7T) compared with high-field (3T) strength. Eur Radiol 2011;21:1136-1143.

21 Hyun JY, Young CY, Bong-Keun C: T2 values of femoral cartilage of the knee joint: comparison between pre-contrast and post-contrast images. Korean J Radiol 2014;15:123-129.

22 Mikko JN, Shabnam M, John H, et al: T2* relaxation time of acetabular and femoral cartilage with and without intra-articular GdDTPA $^{2-}$ in patients with femoroacetabular impingement. AJR Am J Roentgenol 2015; 204:W695-W700.

23 Ligong W, Ravinder RR: Investigation of regional influence of magic-angle effect on T2 in human articular cartilage with osteoarthritis at 3 T. Acad Radiol 2015;22:87-92.

24 Rehnitz C, Kupfer J, Streich NA, et al: Comparison of biochemical cartilage imaging techniques at 3 T MRI. Osteoarthritis Cartilage 2014;22:1732-1742.

25 Raya JG, Dietrich O, Horng A, et al: T2 measurement in articular cartilage: impact of the fitting method on accuracy and precision at low SNR. Magn Reson Med 2010;63:181-193.

26 Koff MF, Amrami KK, Felmlee JP, et al: Bias of cartilage T2 values related to method of calculation. J Magn Reson Imaging 2008;26: 1236-1243.

27 Li X, Benjamin Ma C, Link TM, et al: In vivo T1rho and T2 mapping of articular cartilage in osteoarthritis of the knee using 3 T MRI. Osteoarthritis Cartilage 2007;15:789-797. 